\title{
Schauder bases and the bounded approximation property in separable Banach spaces
}

\author{
by \\ Jorge Mujica and Daniela M. Vieira (Campinas)
}

\begin{abstract}
Let $E$ be a separable Banach space with the $\lambda$-bounded approximation property. We show that for each $\epsilon>0$ there is a Banach space $F$ with a Schauder basis such that $E$ is isometrically isomorphic to a 1-complemented subspace of $F$ and, moreover, the sequence $\left(T_{n}\right)$ of canonical projections in $F$ has the properties

$$
\sup _{n \in \mathbb{N}}\left\|T_{n}\right\| \leq \lambda+\epsilon \quad \text { and } \quad \limsup _{n \rightarrow \infty}\left\|T_{n}\right\| \leq \lambda .
$$

This is a sharp quantitative version of a classical result obtained independently by Pełczyński and by Johnson, Rosenthal and Zippin.
\end{abstract}

Introduction. Pełczyński [12] and Johnson, Rosenthal and Zippin [7] have independently shown that every separable Banach space with the bounded approximation property is topologically isomorphic to a complemented subspace of a Banach space with a Schauder basis.

If $E$ is a separable Banach space with the $\lambda$-bounded approximation property, then the proof of Pełczyński [12, which has been reproduced in the book of Lindenstrauss and Tzafriri [8, p. 38], shows that $E$ is isometrically isomorphic to a 1-complemented subspace of a Banach space $F$ with a Schauder basis, whose basis constant is at most $5 \lambda$.

In this paper we show that if $E$ is a separable Banach space with the $\lambda$-bounded approximation property, then for each $\epsilon>0$ there is a Banach space $F$ with a Schauder basis, whose basis constant is at most $\lambda+\epsilon$, such that $E$ is isometrically isomorphic to a 1-complemented subspace of $F$. Our proof is a refinement of the proof of Pełczyński [12].

We establish our main result in Section 1. In Section 2 we use that result to extend some recent results for holomorphic functions on Banach spaces with Schauder bases (see [11]) to the realm of separable Banach spaces with the bounded approximation property.

2010 Mathematics Subject Classification: Primary 46B15, 46B28, 46G20.

Key words and phrases: Banach space, Schauder basis, bounded approximation property. 
1. Schauder bases and the bounded approximation property. If $\lambda \geq 1$, then a Banach space $E$ is said to have the $\lambda$-bounded approximation property if for each compact set $K \subset E$ and $\epsilon>0$, there is a finite rank operator $T \in L(E ; E)$ such that $\|T\| \leq \lambda$ and $\|T x-x\| \leq \epsilon$ for every $x \in K$; $E$ is said to have the bounded approximation property if $E$ has the $\lambda$-bounded approximation property for some $\lambda \geq 1$; and $E$ is said to have the metric approximation property if $E$ has the 1-bounded approximation property.

A sequence $\left(e_{n}\right) \subset E$ is said to be a Schauder basis if each $x \in E$ can be uniquely written as a convergent series $x=\sum_{n=1}^{\infty} \xi_{n} e_{n}$ with $\left(\xi_{n}\right) \subset \mathbb{K}$. Let $\left(T_{n}\right)$ denote the sequence of canonical projections, that is,

$$
T_{n} x=\sum_{j=1}^{n} \xi_{j} e_{j} \quad \text { for every } x=\sum_{j=1}^{\infty} \xi_{j} e_{j} \in E .
$$

Let $c$ denote the basis constant, and let $c_{a}$ denote the asymptotic basis constant, that is,

$$
c=\sup _{n \in \mathbb{N}}\left\|T_{n}\right\|, \quad c_{a}=\limsup _{n \rightarrow \infty}\left\|T_{n}\right\| .
$$

A Schauder basis is said to be monotone if $c=1$, and asymptotically monotone if $c_{a}=1$. Clearly every monotone basis is asymptotically monotone, but while monotone bases are rather special, asymptotically monotone bases can be found everywhere. Indeed, as pointed out in [11, an examination of the proof of a classical result of Mazur (see [8, Theorem 1.a.5]) shows the following theorem.

THEOREM 1.1. Every infinite-dimensional Banach space contains a closed, infinite-dimensional subspace with an asymptotically monotone Schauder basis.

We remark that Theorem 1.1 was first proved by Day [2]. His proof is based on a generalization of the Borsuk-Ulam antipodal theorem.

We also remark that Szarek [13] has given an example of a separable reflexive Banach space with the metric approximation property which does not have a Schauder basis.

For background information on Schauder bases and approximation properties we refer to the book of Lindenstrauss and Tzafriri [8].

Our main result is the following theorem.

TheOREM 1.2. Let $E$ be a separable Banach space with the $\lambda$-bounded approximation property. Then for each $\epsilon>0$ there is a Banach space $F$ with a Schauder basis such that $E$ is isometrically isomorphic to a 1-complemented subspace of $F$ and, moreover, the sequence $\left(T_{n}\right)$ of canonical projections in $F$ has the properties

$$
c=\sup _{n}\left\|T_{n}\right\| \leq \lambda+\epsilon \quad \text { and } \quad c_{a}=\limsup _{n \rightarrow \infty}\left\|T_{n}\right\| \leq \lambda .
$$


Corollary 1.3. Every separable Banach space with the metric approximation property is isometrically isomorphic to a 1-complemented subspace of a Banach space with an asymptotically monotone Schauder basis.

To prove the theorem we need the following lemma, a refinement of a lemma due to Pełczyński [12].

Lemma 1.4. Let $E$ be a Banach space of finite dimension $n$. Then for each $\epsilon>0$, there are $m \in \mathbb{N}$ and operators $B_{1}, \ldots, B_{m n} \in L(E ; E)$ of rank one such that

$$
\sum_{j=1}^{m n} B_{j} x=x \quad \text { for all } x \in E
$$

and

$$
\left\|\sum_{j=1}^{k} B_{j}\right\|+\left\|\sum_{j=k+1}^{m n} B_{j}\right\| \leq 1+\epsilon \quad \text { for all } 1 \leq k \leq m n .
$$

Proof. By Auerbach's lemma (see [8, Proposition 1.c.3]) there are normone vectors $e_{1}, \ldots, e_{n} \in E$ and $\phi_{1}, \ldots, \phi_{n} \in E^{\prime}$ such that $\phi_{j}\left(e_{k}\right)=\delta_{j k}$ for $j, k=1, \ldots, n$. Let $A_{1}, \ldots, A_{n} \in L(E ; E)$ be defined by $A_{j} x=\phi_{j}(x) e_{j}$. Then each $A_{j}$ has norm one and rank one, and

$$
\sum_{j=1}^{n} A_{j} x=\sum_{j=1}^{n} \phi_{j}(x) e_{j}=x \quad \text { for every } x \in E .
$$

Fix $m \in \mathbb{N}$. Then each integer $k$ with $1 \leq k \leq m n$ can be uniquely written in the form

$$
k=r n+s \quad \text { with } 0 \leq r \leq m-1,1 \leq s \leq n .
$$

If $k$ is given by (2) we define $B_{k}=m^{-1} A_{s}$. By using (1), it follows that

$$
\begin{aligned}
\sum_{j=1}^{k} B_{j} x & =\sum_{j=1}^{r n} B_{j} x+\sum_{j=r n+1}^{r n+s} B_{j} x \\
& =\frac{r}{m} \sum_{t=1}^{n} A_{t} x+\frac{1}{m} \sum_{t=1}^{s} A_{t} x=\frac{r}{m} x+\frac{1}{m} \sum_{t=1}^{s} A_{t} x .
\end{aligned}
$$

Similarly we have

$$
\begin{aligned}
\sum_{j=k+1}^{m n} B_{j} x & =\sum_{j=r n+s+1}^{(r+1) n} B_{j} x+\sum_{j=(r+1) n+1}^{m n} B_{j} x \\
= & \frac{1}{m} \sum_{t=s+1}^{n} A_{t} x+\frac{m-r-1}{m} \sum_{t=1}^{n} A_{t} x=\frac{1}{m} \sum_{t=s+1}^{n} A_{t} x+\frac{m-r-1}{m} x .
\end{aligned}
$$


Since $m n=(m-1) n+n$, it follows that

$$
\sum_{j=1}^{m n} B_{j} x=\frac{m-1}{m} x+\frac{1}{m} x=x .
$$

On the other hand, if $1 \leq k \leq m n$, then

$$
\left\|\sum_{j=1}^{k} B_{j}\right\|+\left\|\sum_{j=k+1}^{m n} B_{j}\right\| \leq \frac{r+s}{m}+\frac{(n-s)+(m-r-1)}{m}=\frac{m+n-1}{m} .
$$

To complete the proof it suffices to choose $m$ such that $(n-1) / m<\epsilon$.

Proof of Theorem 1.2. Let $\left(x_{n}\right)$ be a countable dense subset of $E$. Let $\left(U_{n}\right)$ be a sequence of finite rank operators in $L(E ; E)$ such that $\left\|U_{n}\right\| \leq \lambda$ for every $n$ and $\left\|U_{n} x_{j}-x_{j}\right\| \leq 1 / n$ whenever $1 \leq j \leq n$. It follows that $\left(U_{n}\right)$ converges to the identity uniformly on compact subsets of $E$. If we define $V_{1}=U_{1}$ and $V_{n}=U_{n}-U_{n-1}$ for every $n \geq 2$, then $\left(V_{n}\right)$ is a sequence of finite rank operators in $L(E ; E)$ such that $\sum_{j=1}^{n} V_{j} x=U_{n} x$, and therefore $\sum_{j=1}^{\infty} V_{j} x=x$ for every $x \in E$.

Let $M_{n}=V_{n}(E)$ for every $n$, and let $\left(\epsilon_{n}\right)$ be a strictly decreasing sequence of positive numbers tending to zero, with $\epsilon_{1}=\epsilon / \lambda$. By Lemma 1.4 for each $n \in \mathbb{N}$ there are operators $B_{1}^{n}, \ldots, B_{p_{n}}^{n} \in L\left(M_{n} ; M_{n}\right)$, of rank one, such that

$$
\sum_{j=1}^{p_{n}} B_{j}^{n} x=x \quad \text { for every } x \in M_{n}
$$

and

$$
\left\|\sum_{j=1}^{k} B_{j}^{n}\right\|+\left\|\sum_{j=k+1}^{p_{n}} B_{j}^{n}\right\| \leq 1+\epsilon_{n} \quad \text { for every } 1 \leq k \leq p_{n} .
$$

Set $p_{0}=0$. Then each $k \in \mathbb{N}$ can be uniquely written in the form

$$
k=p_{0}+p_{1}+\cdots+p_{n-1}+s \quad \text { with } 1 \leq s \leq p_{n} .
$$

If $k$ is given by (3), then we define $\delta_{k}=\epsilon_{n}$ and $A_{k}=B_{s}^{n} \circ V_{n}$. Then

$$
\begin{aligned}
\sum_{j=1}^{k} A_{j} x & =\sum_{j=1}^{p_{0}+\cdots+p_{n-1}} A_{j} x+\sum_{j=p_{0}+\cdots+p_{n-1}+1}^{p_{0}+\cdots+p_{n-1}+s} A_{j} x \\
& =\sum_{r=1}^{n-1} \sum_{t=1}^{p_{r}} B_{t}^{r} \circ V_{r} x+\sum_{t=1}^{s} B_{t}^{n} \circ V_{n} x=\sum_{r=1}^{n-1} V_{r} x+\sum_{t=1}^{s} B_{t}^{n} \circ V_{n} x \\
& =U_{n-1} x+\left(\sum_{t=1}^{s} B_{t}^{n}\right) \circ\left(U_{n}-U_{n-1}\right) x \\
& =\left(\sum_{t=1}^{s} B_{t}^{n}\right) \circ U_{n} x+\left(\sum_{t=s+1}^{p_{n}} B_{t}^{n}\right) \circ U_{n-1} x .
\end{aligned}
$$


On the one hand, it follows that

$$
\begin{aligned}
\left\|\sum_{j=1}^{k} A_{j} x-x\right\| & \leq\left\|U_{n-1} x-x\right\|+\left\|\sum_{t=1}^{s} B_{t}^{n}\right\|\left\|\left(U_{n}-U_{n-1}\right) x\right\| \\
& \leq\left\|U_{n-1} x-x\right\|+\left(1+\epsilon_{n}\right)\left\|\left(U_{n}-U_{n-1}\right) x\right\|,
\end{aligned}
$$

and the last expression tends to zero when $n$ tends to infinity. Since each $A_{j}$ has rank one, we may write $A_{j} x=\phi_{j}(x) a_{j}$ with $\phi_{j} \in E^{\prime}$ and $a_{j} \in E$, $\left\|a_{j}\right\|=1$. Thus

$$
x=\sum_{j=1}^{\infty} A_{j} x=\sum_{j=1}^{\infty} \phi_{j}(x) a_{j} \quad \text { for every } x \in E .
$$

On the other hand, it follows that

$$
\left\|\sum_{j=1}^{k} A_{j}\right\| \leq\left\|\sum_{t=1}^{s} B_{t}^{n}\right\|\left\|U_{n}\right\|+\left\|\sum_{t=s+1}^{p_{n}} B_{t}^{n}\right\|\left\|U_{n-1}\right\| \leq \lambda\left(1+\epsilon_{n}\right),
$$

and therefore

$$
\left\|\sum_{j=1}^{k} A_{j}\right\| \leq \lambda\left(1+\delta_{k}\right) \quad \text { for every } k \in \mathbb{N} .
$$

Let $F$ be the vector space of all sequences $y=\left(\eta_{j}\right) \subset \mathbb{K}$ such that the series $\sum_{j=1}^{\infty} \eta_{j} a_{j}$ converges in $E$. Then a standard argument shows that $F$, endowed with the norm

$$
\|y\|=\sup _{k}\left\|\sum_{j=1}^{k} \eta_{j} a_{j}\right\|
$$

is a Banach space whose unit vectors form a monotone Schauder basis. Let $\left(T_{k}\right)$ denote the sequence of canonical projections in $F$, that is,

$$
T_{k} y=\left(\eta_{1}, \ldots, \eta_{k}, 0,0, \ldots\right) \quad \text { for every } y=\left(\eta_{j}\right) \in F \text {. }
$$

Let $A \in L(E ; F)$ and $B \in L(F ; E)$ be defined by

$$
A x=\left(\phi_{j}(x)\right) \text { and } \quad B y=\sum_{j=1}^{\infty} \eta_{j} a_{j} .
$$

Then

$$
\begin{aligned}
\|A x\| & =\sup _{k}\left\|\sum_{j=1}^{k} \phi_{j}(x) a_{j}\right\|=\sup _{k}\left\|\sum_{j=1}^{k} A_{j} x\right\| \\
& \leq \sup _{k} \lambda\left(1+\delta_{k}\right)\|x\|=\lambda\left(1+\epsilon_{1}\right)\|x\|,
\end{aligned}
$$


and

$$
\|B y\|=\left\|\sum_{j=1}^{\infty} \eta_{j} a_{j}\right\| \leq \sup _{k}\left\|\sum_{j=1}^{k} \eta_{j} a_{j}\right\|=\|y\| .
$$

Since $B \circ A x=x$ for every $x \in E, E$ is isomorphic to a complemented subspace of $F$.

Next we will renorm $F$ so as to obtain all the stated properties. Consider the set

$$
W=\left\{y=\left(\eta_{j}\right) \in F:\left\|\sum_{j=1}^{\infty} \eta_{j} a_{j}\right\| \leq 1,\left\|\sum_{j=1}^{k} \eta_{j} a_{j}\right\| \leq \lambda\left(1+\delta_{k}\right) \text { for every } k\right\} .
$$

Clearly $W$ is convex and balanced. Since

$$
W \subset\left\{y=\left(\eta_{j}\right) \in F:\|y\| \leq \lambda\left(1+\epsilon_{1}\right)\right\},
$$

it follows that $W$ is bounded in $F$. And since

$$
W \supset\left\{y=\left(\eta_{j}\right) \in F:\|B y\| \leq 1 \text { and }\|y\| \leq \lambda\right\},
$$

it follows that $W$ is a 0 -neighborhood in $F$, and is in particular absorbing. Let $F_{W}$ denote the vector space $F$, seminormed by the Minkowski functional $p_{W}$ of $W$. Since $W$ is bounded in $F, p_{W}$ is a norm and the identity mapping $F_{W} \rightarrow F$ is continuous. And since $W$ is a 0 -neighborhood in $F$, the identity mapping $F_{W} \rightarrow F$ is actually a homeomorphism. In particular, $F_{W}$ is a Banach space whose unit vectors form a Schauder basis.

If $x \in B_{E}$, then $A x=\left(\phi_{j}(x)\right) \in W$, since

$$
\left\|\sum_{j=1}^{\infty} \phi_{j}(x) a_{j}\right\| \leq 1 \quad \text { and } \quad\left\|\sum_{j=1}^{k} \phi_{j}(x) a_{j}\right\| \leq \lambda\left(1+\delta_{k}\right) \quad \text { for every } k .
$$

Conversely, if $A x=\left(\phi_{j}(x)\right) \in W$, then $x \in B_{E}$, since $\|x\|=\left\|\sum_{j=1}^{\infty} \phi_{j}(x) a_{j}\right\|$ $\leq 1$. Thus $A: E \rightarrow F_{W}$ is an isometric embedding.

Clearly $A \circ B$ is a projection from $F_{W}$ onto $A(E)$. To show that $A(E)$ is a 1-complemented subspace of $F_{W}$ we will show that $A \circ B(y) \in W$ for each $y \in W$. Let $y=\left(\eta_{j}\right) \in W$. Then $A \circ B(y)=\left(\phi_{j}(B y)\right)$. On the one hand,

$$
\left\|\sum_{j=1}^{\infty} \phi_{j}(B y) a_{j}\right\|=\|B(A \circ B(y))\|=\|B y\|=\left\|\sum_{j=1}^{\infty} \eta_{j} a_{j}\right\| \leq 1 .
$$

On the other hand,

$$
\left\|\sum_{j=1}^{k} \phi_{j}(B y) a_{j}\right\|=\left\|\sum_{j=1}^{k} A_{j}(B y)\right\| \leq\left\|\sum_{j=1}^{k} A_{j}\right\|\|B y\| \leq \lambda\left(1+\delta_{k}\right)
$$

for every $k$, proving that $A \circ B(y) \in W$. 
To complete the proof of the theorem we will prove that

$$
T_{k} y \in \lambda\left(1+\delta_{k}\right) W \quad \text { for all } y \in W \text { and } k \in \mathbb{N} .
$$

To see this write $y=\left(\eta_{j}\right)$ and $T_{k} y=\left(\sigma_{j}\right)$, where $\sigma_{j}=\eta_{j}$ if $1 \leq j \leq k$ and $\sigma_{j}=0$ if $j>k$. On the one hand,

$$
\left\|\sum_{j=1}^{\infty} \sigma_{j} a_{j}\right\|=\left\|\sum_{j=1}^{k} \eta_{j} a_{j}\right\| \leq \lambda\left(1+\delta_{k}\right) .
$$

On the other hand, if $1 \leq l \leq k$, then

$$
\left\|\sum_{j=1}^{l} \sigma_{j} a_{j}\right\|=\left\|\sum_{j=1}^{l} \eta_{j} a_{j}\right\| \leq \lambda\left(1+\delta_{l}\right) \leq \lambda\left(1+\delta_{k}\right) \lambda\left(1+\delta_{l}\right) .
$$

Finally, if $l>k$, then

$$
\left\|\sum_{j=1}^{l} \sigma_{j} a_{j}\right\|=\left\|\sum_{j=1}^{k} \eta_{j} a_{j}\right\| \leq \lambda\left(1+\delta_{k}\right) \leq \lambda\left(1+\delta_{k}\right) \lambda\left(1+\delta_{l}\right) .
$$

Thus (4) shows that $\left\|T_{k}\right\| \leq \lambda\left(1+\delta_{k}\right)$ if we regard $T_{k}$ as a member of $L\left(F_{W} ; F_{W}\right)$. It follows that

$$
\sup _{k}\left\|T_{k}\right\| \leq \lambda\left(1+\epsilon_{1}\right)=\lambda+\epsilon \text { and } \quad \limsup _{k \rightarrow \infty}\left\|T_{k}\right\| \leq \lambda,
$$

thus completing the proof.

Johnson [6] has proved that every separable reflexive Banach space with the bounded approximation property is isomorphic to a complemented subspace of a reflexive Banach space with a Schauder basis, but he gives no estimate of the size of the basis constant. On the other hand, Grothendieck [5. Chapitre I, p. 181] (see also [8, Theorem 1.e.15]) has shown that every reflexive Banach space with the approximation property already has the metric approximation property.

Motivated by these results, we would like to prove that every separable reflexive Banach space with the (metric) approximation property is isometrically isomorphic to a 1-complemented subspace of a reflexive Banach space with an asymptotically monotone Schauder basis. But we have been unable to use the method of proof of Theorem 1.2 to prove this.

2. Weakly continuous holomorphic functions. In a recent paper we have made a detailed study of certain algebras of weakly continuous holomorphic functions on a Banach space with a Schauder basis (see [11]). With the aid of Theorem 1.2 we can extend some of those results to the realm of separable Banach spaces with the bounded approximation property.

Before stating our results we have to introduce some notation and terminology. For background information on infinite-dimensional complex analysis we refer to the books of Dineen [3] or Mujica [10]. 
Let $E$ be a complex Banach space, let $U$ be an open subset of $E$, and let $\mathcal{H}(U)$ denote the algebra of all complex-valued holomorphic functions on $U$.

Given $A \subset U$ and $\mathcal{F} \subset \mathcal{H}(U)$, the set $\hat{A}_{\mathcal{F}}$ is defined by

$$
\hat{A}_{\mathcal{F}}=\left\{x \in U:|f(x)| \leq \sup _{A}|f| \text { for every } f \in \mathcal{F}\right\} .
$$

For each $x \in U$ let $d_{U}(x)$ denote the distance from $x$ to the boundary of $U$. For each $A \subset U$ we define $d_{U}(A)=\inf _{x \in A} d_{U}(x)$. The open set $U$ is said to be pseudoconvex if the function $-\log d_{U}$ is plurisubharmonic on $U$.

Let $F$ be a separable Banach space with the $\lambda$-bounded approximation property. By Theorem 1.2 we may identify $F$ with a 1-complemented subspace of a Banach space $E$ with a Schauder basis, whose asymptotic constant is at most $\lambda$. Let $\left(T_{n}\right)$ denote the sequence of canonical projections in $E$, and let $\pi$ be a norm-one projection from $E$ onto $F$.

If $V$ is a pseudoconvex open subset of $F$, then $U=\pi^{-1}(V)$ is a pseudoconvex open subset of $E$ and $U \cap F=V$ (see [10, Proposition 37.8]).

Consider the following sequences of open subsets of $U$ :

$$
\begin{aligned}
& A_{j}(U)=\left\{x \in U: \sup _{n \geq j}\left\|T_{n} x-x\right\|<d_{U}(x)\right\}, \\
& B_{j}(U)=\left\{x \in A_{j}(U):\|x\|<j \text { and } d_{A_{j}(U)}(x)>1 / j\right\}, \\
& C_{j}(U)=\left\{x \in B_{j}(U): \sup _{n \geq j}\left\|T_{n} x-x\right\|<d_{B_{j}(U)}(x)\right\} .
\end{aligned}
$$

Let $\mathcal{C}(U)$ denote the sequence $\left(C_{j}(U)\right)$, and let $\mathcal{H}_{w u}(\mathcal{C}(U))$ denote the subalgebra of all $f \in \mathcal{H}(U)$ that are weakly uniformly continuous on each $C_{j}(U)$. It is a Fréchet algebra for the topology of uniform convergence on the sets $C_{j}(U)$.

Next consider the following sequence of open subsets of $V$ :

$$
C_{j}(V)=C_{j}(U) \cap F \subset U \cap F=V .
$$

Let $\mathcal{C}(V)$ denote the sequence $\left(C_{j}(V)\right)$, and let $\mathcal{H}_{w u}(\mathcal{C}(V))$ denote the subalgebra of all $f \in \mathcal{H}(V)$ that are weakly uniformly continuous on each $C_{j}(V)$. It is a Fréchet algebra for the topology of uniform convergence on the sets $C_{j}(V)$.

With this terminology we have the following theorem.

Theorem 2.1. (a) Let $E$ be a Banach space with a Schauder basis, and let $U$ be a pseudoconvex open subset of $E$. Then $U$ is the domain of existence of a function $f \in \mathcal{H}_{w u}(\mathcal{C}(U))$.

(b) Let $F$ be a separable Banach space with the bounded approximation property, and let $V$ be a pseudoconvex open subset of $F$. Then $V$ is the domain of existence of a function $g \in \mathcal{H}_{w u}(\mathcal{C}(V))$. 
Proof. (a) was proved in [11, Theorem 1.2], and the proof rests on the following property of the sequence $\left(C_{j}(U)\right)$, established in [11, Lemma 1.4]:

$$
d_{U}\left({\widehat{C_{j}(U)}}_{\mathcal{H}_{w u}(\mathcal{C}(U))}\right)>0 \quad \text { for every } j
$$

(b) The proof of (b) is a straightforward adaptation of the proof of [11, Theorem 1.2], and the proof rests on the following property of the sequence $\left(C_{j}(V)\right)$ :

$$
d_{V}\left({\widehat{C_{j}(V)}}_{\mathcal{H}_{w u}(\mathcal{C}(V))}\right)>0 \quad \text { for every } j .
$$

We can readily see that $(6)$ follows from (5). Indeed, if ${\widehat{C_{j}(U)}}_{\mathcal{H}_{w u}(\mathcal{C}(U))}+$ $B_{E}(0 ; r) \subset U$, then, since $\mathcal{H}_{w u}(\mathcal{C}(U)) \subset \mathcal{H}_{w u}(\mathcal{C}(V))$, it follows that ${\widehat{C_{j}(V)}}_{\mathcal{H}_{w u}(\mathcal{C}(V))}+B_{F}(0 ; r) \subset{\widehat{C_{j}(U)}}_{\mathcal{H}_{w u}(\mathcal{C}(U))} \cap F+B_{E}(0 ; r) \cap F \subset U \cap F=V$.

Let $0<\alpha \leq 1$, and let $\mathcal{H}_{\alpha w u d}(U)$ (resp. $\mathcal{H}_{\alpha d}(U)$ ) denote the algebra of all $f \in \mathcal{H}(U)$ that are weakly uniformly continuous (resp. bounded) on each ball $B(x ; r)$ with $x \in U$ and $0<r<\alpha d_{U}(x)$. It follows from an argument of Aron and Prolla [1, Lemma 2.2] that $\mathcal{H}_{\alpha w u d}(U) \subset \mathcal{H}_{\alpha d}(U)$. If the underlying space is separable, then $\mathcal{H}_{\alpha d}(U)$ is a Fréchet space for the topology of uniform convergence on the balls $B(x ; r)$ with $x \in U$ and $0<r<\alpha d_{U}(x)$, and $\mathcal{H}_{\alpha w u d}(U)$ is a closed subalgebra of $\mathcal{H}_{\alpha d}(U)$. The algebra $\mathcal{H}_{\alpha d}(U)$ was studied by Matos [9] a long time ago, and has recently been rediscovered by Dineen and Venkova [4] when $\alpha=1$. With this terminology we have the following theorem.

TheOREM 2.2. (a) Let $E$ be a Banach space with a Schauder basis, and let $U$ be a pseudoconvex open subset of $E$. If $\alpha=\left(3 c_{a}\right)^{-2}$, then $U$ is the domain of existence of a function $f \in \mathcal{H}_{\text {awud }}(U)$.

(b) Let $F$ be a separable Banach space with the $\lambda$-bounded approximation property, and let $V$ be a pseudoconvex open subset of $F$. If $\beta=(3 \lambda)^{-2}$, then $V$ is the domain of existence of a function $g \in \mathcal{H}_{\beta w u d}(V)$.

Proof. (a) was proved in [11, Theorem 1.5], and the proof rests on the following property of the sequence $\left(C_{j}(U)\right)$, established in [11, Lemma 1.6]:

(7) For each $x \in U$ and $0<r<\left(3 c_{a}\right)^{-2} d_{U}(x)$, the ball $B_{E}(x, r)$ is contained in some $C_{j}(U)$.

If $\alpha=\left(3 c_{a}\right)^{-2}$, then it follows from (7) that $\mathcal{H}_{w u}(\mathcal{C}(U)) \subset \mathcal{H}_{\text {owud }}(U)$, and then (a) follows from Theorem 2.1(a).

(b) The proof of (b) is similar. Indeed, it follows from Theorem 1.2 that $c_{a} \leq \lambda$, and therefore (7) implies the following:

(8) For each $y \in V$ and $0<r<(3 \lambda)^{-2} d_{V}(y)$, the ball $B_{F}(y ; r)$ is contained in some $C_{j}(V)$. 
If $\beta=(3 \lambda)^{-2}$, then it follows from (8) that $\mathcal{H}_{w u}(\mathcal{C}(V)) \subset \mathcal{H}_{\beta w u d}(V)$, and then (b) follows from Theorem 2.1(b).

TheOREM 2.3. (a) Let $E$ be a Banach space with a Schauder basis, and let $U$ be a pseudoconvex open subset of $E$. Let $\left(x_{p}\right)$ be a sequence of distinct points of $U$ such that $d_{U}\left(x_{p}\right) \rightarrow 0$. Then there exists $f \in \mathcal{H}_{w u}(\mathcal{C}(U))$ such that $\left|f\left(x_{p}\right)\right| \rightarrow \infty$ and $f\left(x_{p}\right) \neq f\left(x_{q}\right)$ whenever $p \neq q$.

(b) Let $F$ be a separable Banach space with the bounded approximation property, and let $V$ be a pseudoconvex open subset of $F$. Let $\left(y_{p}\right)$ be a sequence of distinct points of $V$ such that $d_{V}\left(y_{p}\right) \rightarrow 0$. Then there exists $g \in \mathcal{H}_{w u}(\mathcal{C}(V))$ such that $\left|g\left(y_{p}\right)\right| \rightarrow \infty$ and $g\left(y_{p}\right) \neq g\left(y_{q}\right)$ whenever $p \neq q$.

Proof. (a) was proved in [11, Theorem 2.1], and the proof rests on property (5) of the sequence $\left(C_{j}(U)\right)$.

(b) The proof of (b) is a straightforward adaptation of the proof of 11, Theorem 2.1], and rests on property $(6)$ of $\left(C_{j}(V)\right)$.

TheOREM 2.4. (a) Let $E$ be a Banach space with a Schauder basis, and let $U$ be a pseudoconvex open subset of $E$. Let $\left(x_{p}\right)$ be a sequence of distinct points of $U$ such that $d_{U}\left(x_{p}\right) \rightarrow 0$. If $\alpha=\left(3 c_{a}\right)^{-2}$, then there exists $f \in$ $\mathcal{H}_{\text {owud }}(U)$ such that $\left|f\left(x_{p}\right)\right| \rightarrow \infty$ and $f\left(x_{p}\right) \neq f\left(x_{q}\right)$ whenever $p \neq q$.

(b) Let $F$ be a separable Banach space with the $\lambda$-bounded approximation property, and let $V$ be a pseudoconvex open subset of $F$. Let $\left(y_{p}\right)$ be a sequence of distinct points of $V$ such that $d_{V}\left(y_{p}\right) \rightarrow 0$. If $\beta=(3 \lambda)^{-2}$, then there exists $g \in \mathcal{H}_{\beta w u d}(V)$ such that $\left|g\left(y_{p}\right)\right| \rightarrow \infty$ and $g\left(y_{p}\right) \neq g\left(y_{q}\right)$ whenever $p \neq q$.

Proof. (a) was proved in [11, Theorem 2.3]. Actually, it follows from property (7) of the sequence $\left(C_{j}(U)\right)$ that $\mathcal{H}_{w u}(\mathcal{C}(U)) \subset \mathcal{H}_{\alpha w u d}(U)$, and then (a) follows from Theorem 2.3(a).

(b) Likewise, it follows from property (8) of the sequence $\left(C_{j}(V)\right)$ that $\mathcal{H}_{w u}(\mathcal{C}(V)) \subset \mathcal{H}_{\beta w u d}(V)$, and then (b) follows from Theorem 2.3(b).

If $G$ is a Banach space, then the space $\mathcal{H}_{\alpha w u d}(U ; G)$ is defined in the obvious way.

TheOREM 2.5. (a) Let $E$ be a Banach space with a Schauder basis, and let $U$ be a pseudoconvex open subset of $E$. Let $\left(x_{p}\right)$ be a sequence of distinct points of $U$ such that $d_{U}\left(x_{p}\right) \rightarrow 0$, and let $\left(z_{p}\right)$ be an arbitrary sequence in a Banach space $G$. If $\alpha=\left(3 c_{a}\right)^{-2}$, then there exists $f \in \mathcal{H}_{\alpha w u d}(U ; G)$ such that $f\left(x_{p}\right)=z_{p}$ for every $p$.

(b) Let $F$ be a separable Banach space with the $\lambda$-bounded approximation property, and let $V$ be a pseudoconvex open subset of $F$. Let $\left(y_{p}\right)$ be a sequence of distinct points of $V$ such that $d_{V}\left(y_{p}\right) \rightarrow 0$, and let $\left(z_{p}\right)$ be an arbitrary sequence in a Banach space $G$. If $\beta=(3 \lambda)^{-2}$, then there exists $g \in \mathcal{H}_{\beta w u d}(V ; G)$ such that $g\left(y_{p}\right)=z_{p}$ for every $p$. 
Proof. (a) was proved in [11, Theorem 2.6]. Actually, it follows from Theorem 2.4(a) and [11, Lemma 2.5].

(b) Likewise (b) follows from Theorem 2.4(b) and [11, Lemma 2.5].

$\tau_{0}$ denotes the compact-open topology on $\mathcal{H}(U)$.

TheOREM 2.6. (a) Let $E$ be a Banach space with a Schauder basis, and let $U$ be a pseudoconvex open subset of E. If $\alpha=\left(3 c_{a}\right)^{-2}$, then $\mathcal{H}_{\alpha w u d}(U)$ is sequentially dense in $\left(\mathcal{H}(U), \tau_{0}\right)$.

(b) Let $F$ be a separable Banach space with the $\lambda$-bounded approximation property, and let $V$ be a pseudoconvex open subset of $F$. If $\beta=(3 \lambda)^{-2}$, then $\mathcal{H}_{\beta w u d}(V)$ is sequentially dense in $\left(\mathcal{H}(V), \tau_{0}\right)$.

Proof. (a) was proved in a more general form in [11, Theorem 3.2].

(b) By Theorem 1.2 we may identify $F$ with a 1-complemented subspace of a Banach space $E$ with a Schauder basis, with $c_{a} \leq \lambda$. Let $\pi$ be a normone projection from $E$ onto $F$, and let $U=\pi^{-1}(V)$. If $g \in \mathcal{H}(V)$, then $g \circ \pi \in \mathcal{H}(U)$. By (a) there is a sequence $\left(f_{n}\right) \subset \mathcal{H}_{\text {awud }}(U)$ which converges to $g \circ \pi$ in $\left(\mathcal{H}(U), \tau_{0}\right)$. If we define $g_{n}=f_{n}\left|U \cap F=f_{n}\right| V$, then it follows that $\left(g_{n}\right) \subset \mathcal{H}_{\text {awud }}(V)$ and $\left(g_{n}\right)$ converges to $g$ in $\left(\mathcal{H}(V), \tau_{0}\right)$. Since $c_{a} \leq \lambda$, it follows that $\beta \leq \alpha$, and the proof is complete.

In a similar manner we could extend other results from [11] to the realm of separable Banach spaces with the bounded approximation property.

Acknowledgements. This research was supported by FAPESP, Brazil, Project 06/02378-7.

\section{References}

[1] R. Aron and J. B. Prolla, Polynomial approximation of differentiable functions on Banach spaces, J. Reine Angew. Math. 313 (1980), 195-216.

[2] M. M. Day, On the basis problem in normed spaces, Proc. Amer. Math. Soc. 13 (1962), 655-658.

[3] S. Dineen, Complex Analysis on Infinite Dimensional Spaces, Springer, London, 1999.

[4] S. Dineen and M. Venkova, Extending bounded type holomorphic mappings on a Banach space, J. Math. Anal. Appl. 297 (2004), 645-658.

[5] A. Grothendieck, Produits tensoriels topologiques et espaces nucléaires, Mem. Amer. Math. Soc. 16 (1955).

[6] W. B. Johnson, Factoring compact operators, Israel J. Math. 9 (1971), 337-345.

[7] W. B. Johnson, H. P. Rosenthal and M. Zippin, On bases, finite dimensional decompositions and weaker structures in Banach spaces, ibid. 9 (1971), 488-506.

[8] J. Lindenstrauss and L. Tzafriri, Classical Banach Spaces I, Springer, Berlin, 1977.

[9] M. Matos, Domains of $\tau$-holomorphy in a separable Banach space, Math. Ann. 195 (1972), 273-278.

[10] J. Mujica, Complex Analysis in Banach Spaces, North-Holland Math. Stud. 120, North-Holland, Amsterdam, 1986. 
[11] J. Mujica and D. M. Vieira, Weakly continuous holomorphic functions on pseudoconvex domains in Banach spaces, preprint.

[12] A. Pełczyński, Any separable Banach space with the bounded approximation property is a complemented subspace of a Banach space with a basis, Studia Math. 40 (1971), 239-243.

[13] S. Szarek, A Banach space without a basis which has the bounded approximation property, Acta Math. 159 (1987), 81-98.

Jorge Mujica, Daniela M. Vieira

IMECC-UNICAMP

Caixa Postal 6065

13083-970 Campinas, SP, Brazil

E-mail: mujica@ime.unicamp.br danim@ime.unicamp.br
Current address of Daniela M. Vieira: Instituto de Matemática e Estatística Universidade de São Paulo Caixa Postal 66281 05315-970 São Paulo, SP, Brazil E-mail: danim@ime.usp.br

Received August 30, 2007

Revised version November 26, 2008 